\title{
Solving dominance and potential optimality in imprecise multi-attribute additive problems
}

\author{
Alfonso Mateos*, Antonio Jiménez, Sixto Ríos-Insua \\ Department of Artificial Intelligence, School of Computer Science, Madrid Technical University, Campus de Montegancedo s/n, \\ 28660 Boadilla del Monte, Madrid, Spain
}

\begin{abstract}
We consider the multicriteria decision-making problem where there is partial information on decision maker preferences, represented by means of an imprecise multiattribute additive utility function, and where the consequences of the alternatives or strategies are also possibly imprecise. Under these circumstances we consider how useful problem-solving concepts, namely nondominated, potentially optimal, adjacent potentially optimal alternatives, can be analytically computed. Thus, the problem can be solved much more efficiently using the classical methodology of linear programming.
\end{abstract}

\section{Introduction}

The traditional approach to decision analysis calls for single or precise values for the different model inputs, i.e. for the weight and utility assessments, as well as for the multiattributed consequences of the generated alternatives [1-3]. Here, we take an approach that is less demanding on the decision maker (DM), who is allowed to provide ranges (value intervals) instead of single values for the above inputs $[4,5]$. These ranges will be later used to allow for simultaneous variation in all the data, taking advantage of advances in optimization theory to make it easier for the DM to choose an optimal solution. Thus, although it will be possible as to output the ranking of alternatives or decision strategies based on their precise utilities, we present here some computational procedures that exploit all the information about ranges in weights, utilities and consequences, for the purpose of helping the DM to choose the most preferred alternative, as well as to reduce the set of alternatives of interest.

Let us consider the multi-objective decision making problem where the objectives can be represented in an objectives hierarchy $[3,4,6]$ in which there are $n$ lowestlevel objectives each associated with an attribute, Fig. 1, which measures the achievement of each alternative with

\footnotetext{
* Corresponding author.
}

E-mail address: amateos@fi.upm.es. (A. Mateos). respect to each attribute. Thus, a vector or multi-attributed consequences will be associated with each alternative, which have to be ranked.

Suppose that a DM has to choose among a finite set of alternatives $\mathscr{A}=\left\{S_{1}, \ldots, S_{q}\right\}$, of which the multi-attributed consequences are not exactly known. Hence, instead of by a vector with single values as above, the alternatives are defined by means of vectors of intervals associated with each attribute of the hierarchy, which are able to reflect the situation in which there is some uncertainty about the policy effects of the consequences. In this case, the alternatives will be

$$
\begin{aligned}
& S_{1}=\left(\left[x_{m 1}^{1}, x_{M 1}^{1}\right],\left[x_{m 2}^{1}, x_{M 2}^{1}\right], \ldots,\left[x_{m n}^{1}, x_{M n}^{1}\right]\right) \\
& S_{2}=\left(\left[x_{m 1}^{2}, x_{M 1}^{2}\right],\left[x_{m 2}^{2}, x_{M 2}^{2}\right], \ldots,\left[x_{m n}^{2}, x_{M n}^{2}\right]\right) \\
& \vdots \\
& S_{q}=\left(\left[x_{m 1}^{q}, x_{M 1}^{q}\right],\left[x_{m 2}^{q}, x_{M 2}^{q}\right], \ldots,\left[x_{m n}^{q}, x_{M n}^{q}\right]\right)
\end{aligned}
$$

where $x_{m i}^{j}$ and $x_{M i}^{j}$ will be the minimum $(m)$ and maximum $(M)$ values, taken by each alternative $S_{j}$ for the attribute $i$, respectively, with $j=1, \ldots, q$ and $i=1, \ldots, n$.

We consider that the DM preferences can be represented by means of an additive utility function, $[3,7,8]$, which takes the form

$$
u(\cdot)=\sum_{i=1}^{n} w_{i} u_{i}(\cdot)
$$



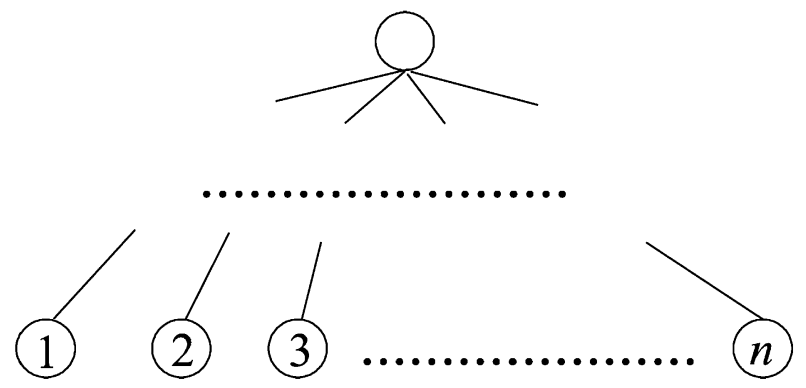

Fig. 1. Generic objectives hierarchy.

where $w_{i}$ and $u_{i}$ are the weight, or scaling factor, and the (individual) utility function for attribute $i(i=1, \ldots, n)$, respectively.

In this context of imprecision, we assume that we also have intervals instead of single or precise values for the weights or scaling factors. Thus, if we suppose that the weight $w_{i} \in\left[w_{m}^{i}, w_{M}^{i}\right]$ for all $i$, where $w_{m}^{i}$ and $w_{M}^{i}$ are the minimum and maximum values for the weight of attribute $i(i=1, \ldots, n)$, the interval weight of each attribute will be obtained by multiplying the ends of the weight interval from the global objective to the attribute under consideration, assessed by means of available weight elicitation methods.

We also allow for imprecise information $[9,10]$ in the assessment procedure of the individual utility functions (probability equivalence/certainty equivalence) [11] obtaining a class of utility functions rather than a unique function for each attribute. Each class of utility functions will be made up of all the utility functions $u_{i}$, comprised between $u_{m i}$ and $u_{M i}$, Fig. 2, where $x_{i}^{m}$ and $x_{i}^{M}$ are the least and most preferred values by the DM for attribute $i, x_{i}^{\prime}, x_{i}^{\prime \prime}$ and $x_{i}^{\prime \prime \prime}$ are three intermediate values for the attribute $i$, and $\left[u_{m i}^{\prime}, u_{M i}^{\prime}\right]$, $\left[u_{m i}^{\prime \prime}, u_{M i}^{\prime \prime}\right]$ and $\left[u_{m i}^{\prime \prime \prime}, u_{M i}^{\prime \prime \prime}\right]$ are the utility intervals that, for the DM, represent the values $x_{i}^{\prime}, x_{i}^{\prime \prime}$ and $x_{i}^{\prime \prime \prime}$, respectively.

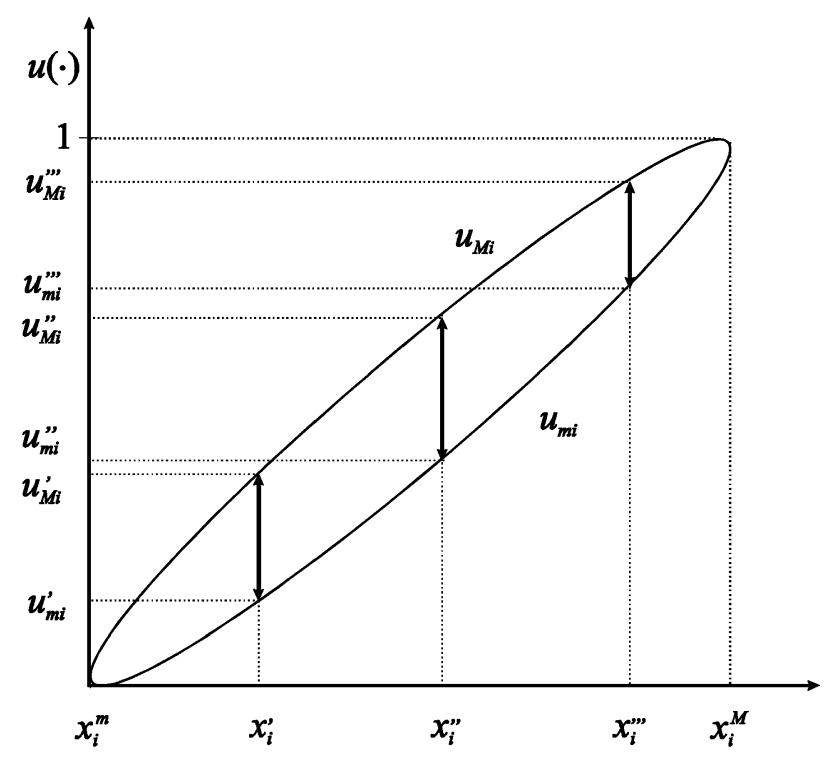

Fig. 2. Class of utility functions for attribute $i$.
The implementation of the imprecise weight and utility assessments combining different methods is explained in detail for a variety of real applications in Refs. [4,5,12]

The functions $u_{m i}$ and $u_{M i}$ are obtained by fitting the cubic splines that go through the points $\left(x_{i}^{m}, 0\right),\left(x_{i}^{\prime}, u_{m i}^{\prime}\right)$, $\left(x_{i}^{\prime \prime}, u_{m i}^{\prime \prime}\right),\left(x_{i}^{\prime \prime \prime}, u_{m i}^{\prime \prime \prime}\right)$ and $\left(x_{i}^{M}, 1\right)$ and the points $\left(x_{i}^{m}, 0\right),\left(x_{i}^{\prime}, u_{M i}^{\prime}\right)$, $\left(x_{i}^{\prime \prime}, u_{M i}^{\prime \prime}\right),\left(x_{i}^{\prime \prime \prime}, u_{M i}^{\prime \prime \prime}\right)$ and $\left(x_{i}^{M}, 1\right)$, respectively. The procedure is as follows. We define a cubic spline for each interval, having then

$$
\begin{aligned}
& {\left[x_{i}^{m}, x_{i}^{\prime}\right] \rightarrow a_{i 1}+b_{i 1} x+c_{i 1}(x)^{2}+d_{i 1}(x)^{3}} \\
& {\left[x_{i}^{\prime}, x_{i}^{\prime \prime}\right] \rightarrow a_{i 2}+b_{i 2} x+c_{i 2}(x)^{2}+d_{i 2}(x)^{3}} \\
& {\left[x_{i}^{\prime \prime}, x_{i}^{\prime \prime \prime}\right] \rightarrow a_{i 3}+b_{i 3} x+c_{i 3}(x)^{2}+d_{i 3}(x)^{3}} \\
& {\left[x_{i}^{\prime \prime \prime}, x_{i}^{M}\right] \rightarrow a_{i 4}+b_{i 4} x+c_{i 4}(x)^{2}+d_{i 4}(x)^{3}}
\end{aligned}
$$

For each attribute $i$, we have 16 unknown quantities, $a_{i j}, b_{i j}$, $c_{i j}, d_{i j}$ with $j=1-4$. We, therefore, need the same number of equations to set out a system and then obtain the cubic splines by intervals. The equations/constraints for obtaining the $u_{m i}$ are

- On the value taken by the utility function at the extremes of the interval.

- If the utility function is increasing:

$$
\begin{aligned}
& a_{i 1}+b_{i 1} x_{i}^{m}+c_{i 1}\left(x_{i}^{m}\right)^{2}+d_{i 1}\left(x_{i}^{m}\right)^{3}=0 \\
& a_{i 4}+b_{i 4} x_{i}^{M}+c_{i 4}\left(x_{i}^{M}\right)^{2}+d_{i 4}\left(x_{i}^{M}\right)^{3}=1
\end{aligned}
$$

- If the utility function is decreasing:

$$
\begin{aligned}
& a_{i 1}+b_{i 1} x_{i}^{M}+c_{i 1}\left(x_{i}^{M}\right)^{2}+d_{i 1}\left(x_{i}^{M}\right)^{3}=1 \\
& a_{i 4}+b_{i 4} x_{i}^{m}+c_{i 4}\left(x_{i}^{m}\right)^{2}+d_{i 4}\left(x_{i}^{m}\right)^{3}=0
\end{aligned}
$$

- On the values taken by the utility function at the intermediate points of the interval:

$$
\begin{aligned}
& a_{i 1}+b_{i 1} x_{i}^{\prime}+c_{i 1}\left(x_{i}^{\prime}\right)^{2}+d_{i 1}\left(x_{i}^{\prime}\right)^{3}=u_{m i}^{\prime} \\
& a_{i 2}+b_{i 2} x_{i}^{\prime \prime}+c_{i 2}\left(x_{i}^{\prime \prime}\right)^{2}+d_{i 2}\left(x_{i}^{\prime \prime}\right)^{3}=u_{m i}^{\prime \prime} \\
& a_{i 3}+b_{i 3} x_{i}^{\prime \prime \prime}+c_{i 3}\left(x_{i}^{\prime \prime \prime}\right)^{2}+d_{i 3}\left(x_{i}^{\prime \prime \prime}\right)^{3}=u_{m i}^{\prime \prime \prime}
\end{aligned}
$$

- On the continuity of the utility function at the intermediate points of the interval:

$$
\begin{aligned}
a_{i 1} & +b_{i 1} x_{i}^{\prime}+c_{i 1}\left(x_{i}^{\prime}\right)^{2}+d_{i 1}\left(x_{i}^{\prime}\right)^{3} \\
& =a_{i 2}+b_{i 2} x_{i}^{\prime}+c_{i 2}\left(x_{i}^{\prime}\right)^{2}+d_{i 2}\left(x_{i}^{\prime}\right)^{3} \\
a_{i 2} & +b_{i 2} x_{i}^{\prime \prime}+c_{i 2}\left(x_{i}^{\prime \prime}\right)^{2}+d_{i 2}\left(x_{i}^{\prime \prime}\right)^{3} \\
& =a_{i 3}+b_{i 3} x_{i}^{\prime \prime}+c_{i 3}\left(x_{i}^{\prime \prime}\right)^{2}+d_{i 3}\left(x_{i}^{\prime \prime}\right)^{3} \\
a_{i 3} & +b_{i 3} x_{i}^{\prime \prime \prime}+c_{i 3}\left(x_{i}^{\prime \prime \prime}\right)^{2}+d_{i 3}\left(x_{i}^{\prime \prime \prime}\right)^{3} \\
& =a_{i 4}+b_{i 4} x_{i}^{\prime \prime \prime}+c_{i 4}\left(x_{i}^{\prime \prime \prime}\right)^{2}+d_{i 4}\left(x_{i}^{\prime \prime \prime}\right)^{3}
\end{aligned}
$$


- On the continuity of the first derivative of the utility function at the intermediate points of the interval:

$$
\begin{aligned}
& b_{i 1}+2 c_{i 1} x_{i}^{\prime}+3 d_{i 1}\left(x_{i}^{\prime}\right)^{2}=b_{i 2}+2 c_{i 2} x_{i}^{\prime}+3 d_{i 2}\left(x_{i}^{\prime}\right)^{2} \\
& b_{i 2}+2 c_{i 2} x_{i}^{\prime \prime}+3 d_{i 2}\left(x_{i}^{\prime \prime}\right)^{2}=b_{i 3}+2 c_{i 3} x_{i}^{\prime \prime}+3 d_{i 3}\left(x_{i}^{\prime \prime}\right)^{2} \\
& b_{i 3}+2 c_{i 3} x_{i}^{\prime \prime \prime}+3 d_{i 3}\left(x_{i}^{\prime \prime \prime}\right)^{2}=b_{i 4}+2 c_{i 4} x_{i}^{\prime \prime \prime}+3 d_{i 4}\left(x_{i}^{\prime \prime \prime}\right)^{2}
\end{aligned}
$$

- On the continuity of the second derivative of the utility function at the intermediate points of the interval:

$$
\begin{aligned}
& 2 c_{i 1}+6 d_{i 1} x_{i}^{\prime}=2 c_{i 2}+6 d_{i 2} x_{i}^{\prime} \\
& 2 c_{i 2}+6 d_{i 2} x_{i}^{\prime \prime}=2 c_{i 3}+6 d_{i 3} x_{i}^{\prime \prime} \\
& 2 c_{i 3}+6 d_{i 3} x_{i}^{\prime \prime \prime}=2 c_{i 4}+6 d_{i 4} x_{i}^{\prime \prime \prime}
\end{aligned}
$$

- On the first derivative of the utility function at the extremes of the interval for the range of each attribute:

- If the utility function is increasing:

$$
\begin{aligned}
& b_{i 1}+2 c_{i 1} x_{i}^{m}+3 d_{i 1}\left(x_{i}^{m}\right)^{2}=0 \\
& b_{i 4}+2 c_{i 4} x_{i}^{M}+3 d_{i 4}\left(x_{i}^{M}\right)^{2}=0
\end{aligned}
$$

- If the utility function is decreasing:

$$
\begin{aligned}
& b_{i 1}+2 c_{i 1} x_{i}^{M}+3 d_{i 1}\left(x_{i}^{M}\right)^{2}=0 \\
& b_{i 4}+2 c_{i 4} x_{i}^{m}+3 d_{i 4}\left(x_{i}^{m}\right)^{2}=0
\end{aligned}
$$

The $u_{M i}$ are obtained by solving the same equations after substituting $u_{m i}^{\prime}, u_{m i}^{\prime \prime}$ and $u_{m i}^{\prime \prime \prime}$ by $u_{M i}^{\prime}, u_{M i}^{\prime \prime}$ and $u_{M i}^{\prime \prime \prime}$, respectively, in Eq. (3). Once the extreme utility functions $u_{m i}$ and $u_{M i}$ have been determined, we know that the condition $u_{m i}(\cdot) \leq u_{i}(\cdot) \leq u_{M i}(\cdot)$ must hold for the true, albeit unknown, utility function $u_{i}$ for attribute $i$.

As we have allowed for imprecision for computing the individual utility functions (obtaining a class of utility functions), we get a value interval, not a single precise value, for the additive multiattribute utility function after computing the weights for the attributes and the consequences of the alternatives on each attribute. The lower limit of this interval is obtained by taking, in each attribute, the lower limit of the value interval of the respective alternative if the utility function is increasing (and the upper limit if it is decreasing), i.e. $x_{m i}^{q}$ (or $x_{M i}^{q}$ ); and taking the function $u_{m i}(\cdot)$ as the utility function of the attribute $i$. We calculate the upper limit of the interval similarly.

Simplistically speaking, we could rank the alternatives using the value obtained when considering the midpoint of the utility interval of each alternative as reference, which should correspond to an approach under precision. However, the underlying idea is to exploit all the above imprecise information to aid the DM in reducing the set of alternatives of interest. To achieve this objective, let us look first at how we could obtain the nondominated strategies and then calculate the potentially optimal and, finally, adjacent potentially optimal to the optimal alternative obtained under precision, [13-16].

\section{The computation of nondominated alternatives}

It is interesting to output the nondominated alternatives because of the following property. If one strategy or alternative dominates another one, this alternative can be discarded as the solution of interest for the DM. Thus, this section focuses on providing a procedure to order the alternatives in a Pareto sense. Given two alternatives $S_{\mathrm{A}}$ and $S_{\mathrm{C}}$, the alternative $S_{\mathrm{A}}$ dominates $S_{\mathrm{C}}$ if $f_{\mathrm{CA}}^{*}>0$, where $f_{\mathrm{CA}}^{*}$ is the optimal value of the optimization problem:

$$
\begin{gathered}
\min f_{\mathrm{CA}}=u\left(S_{\mathrm{A}}\right)-u\left(S_{\mathrm{C}}\right)=\sum_{i=1}^{n} w_{i} u_{i}\left(x_{i}^{\mathrm{A}}\right)-\sum_{i=1}^{n} w_{i} u_{i}\left(x_{i}^{\mathrm{C}}\right) \\
\text { s.t. } \quad \begin{cases}x_{m i}^{\mathrm{A}} \leq x_{i}^{\mathrm{A}} \leq x_{M i}^{\mathrm{A}}, & i=1,2, \ldots, n \\
x_{m i}^{\mathrm{C}} \leq x_{i}^{\mathrm{C}} \leq x_{M i}^{\mathrm{C}}, & i=1,2, \ldots, n \\
w_{m}^{i} \leq w_{i} \leq w_{M}^{i}, & i=1,2, \ldots, n \\
u_{m i}\left(x_{i}^{\mathrm{A}}\right) \leq u_{i}\left(x_{i}^{\mathrm{A}}\right) \leq u_{M i}\left(x_{i}^{\mathrm{A}}\right), & i=1,2, \ldots, n \\
u_{m i}\left(x_{i}^{\mathrm{C}}\right) \leq u_{i}\left(x_{i}^{\mathrm{C}}\right) \leq u_{M i}\left(x_{i}^{\mathrm{C}}\right), & i=1,2, \ldots, n\end{cases}
\end{gathered}
$$

The objective function of the optimization problem (9) is nonlinear, because the variables $w_{i}$ multiply to the variables $a_{i j}, b_{i j}, c_{i j}, d_{i j}$, with $j=1-4$, which correspond to the utility function $u_{i}(\cdot)$. However, all the constraints are linear, although, due to the notation used, they, and specially the last two blocks, do not appear to be at first glance. This is because the constraints

$$
\begin{aligned}
& u_{m i}\left(x_{i}^{\mathrm{A}}\right) \leq u_{i}\left(x_{i}^{\mathrm{A}}\right) \leq u_{M i}\left(x_{i}^{\mathrm{A}}\right) \\
& u_{m i}\left(x_{i}^{\mathrm{C}}\right) \leq u_{i}\left(x_{i}^{\mathrm{C}}\right) \leq u_{M i}\left(x_{i}^{\mathrm{C}}\right)
\end{aligned}
$$

represent constraints (1) or (2)-(7) or (8) if constraints (3) are substituted by

$$
\begin{aligned}
& u_{m i}^{\prime} \leq a_{i 1}+b_{i 1} x_{i}^{\prime}+c_{i 1}\left(x_{i}^{\prime}\right)^{2}+d_{i 1}\left(x_{i}^{\prime}\right)^{3} \\
& u_{m i}^{\prime \prime} \leq a_{i 2}+b_{i 2} x_{i}^{\prime \prime}+c_{i 2}\left(x_{i}^{\prime \prime}\right)^{2}+d_{i 2}\left(x_{i}^{\prime \prime}\right)^{3} \\
& u_{m i}^{\prime \prime \prime} \leq a_{i 3}+b_{i 3} x_{i}^{\prime \prime \prime}+c_{i 3}\left(x_{i}^{\prime \prime \prime}\right)^{2}+d_{i 3}\left(x_{i}^{\prime \prime \prime}\right)^{3} \\
& a_{i 1}+b_{i 1} x_{i}^{\prime}+c_{i 1}\left(x_{i}^{\prime}\right)^{2}+d_{i 1}\left(x_{i}^{\prime}\right)^{3} \leq u_{M i}^{\prime} \\
& a_{i 2}+b_{i 2} x_{i}^{\prime \prime}+c_{i 2}\left(x_{i}^{\prime \prime}\right)^{2}+d_{i 2}\left(x_{i}^{\prime \prime}\right)^{3} \leq u_{M i}^{\prime \prime} \\
& a_{i 3}+b_{i 3} x_{i}^{\prime \prime \prime}+c_{i 3}\left(x_{i}^{\prime \prime \prime}\right)^{2}+d_{i 3}\left(x_{i}^{\prime \prime \prime}\right)^{3} \leq u_{M i}^{\prime \prime \prime}
\end{aligned}
$$

i.e. constraints (10) represent 19 linear constraints. 

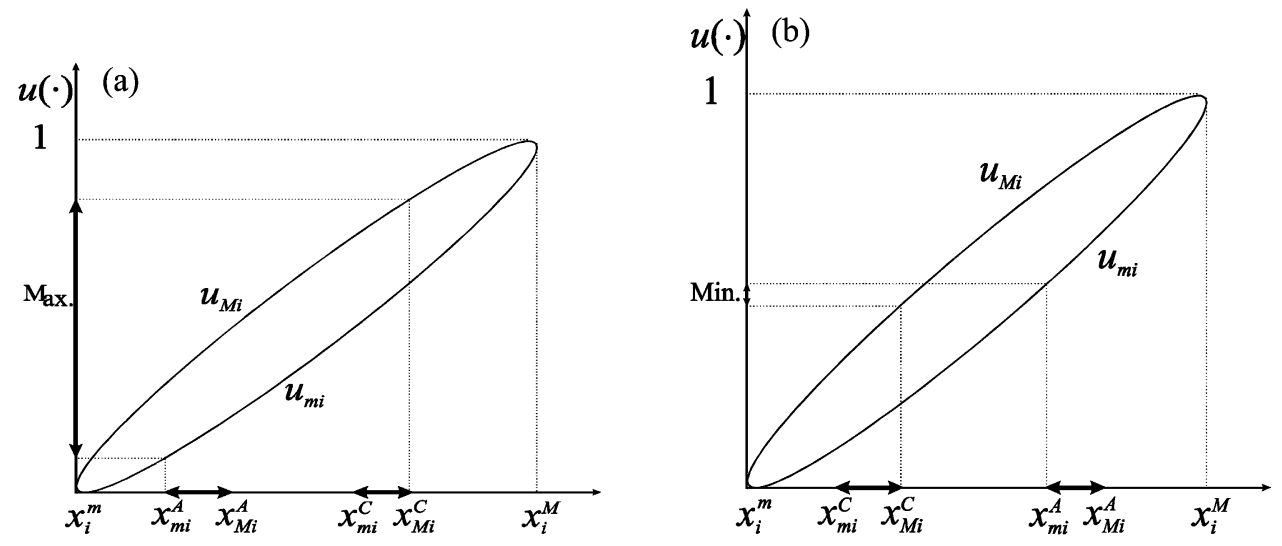

Fig. 3. (a) $S_{\mathrm{C}}$ is preferred to $S_{\mathrm{A}}$; (b) $S_{\mathrm{A}}$ is preferred to $S_{\mathrm{C}}$.

Examining the objective function, we find that it can be rewritten as

$\sum_{i=1}^{n} w_{i}\left[u_{i}\left(x_{i}^{\mathrm{A}}\right)-u_{i}\left(x_{i}^{\mathrm{C}}\right)\right]$

where $u_{i}\left(x_{i}^{\mathrm{A}}\right)-u_{i}\left(x_{i}^{\mathrm{C}}\right)$ does not depend on the weights $w_{i}$. Moreover, if we carefully observe the constraints, we discover that variables $w_{i}$ are independent of the other variables. So, taking into account that the weights $w_{i}$ are nonnegative, solving problem (9) is equivalent to solving the optimization problem

$\min f_{\mathrm{CA}}=\sum_{i=1}^{n} w_{i} z_{i}^{*}$

s.t. $\quad w_{m}^{i} \leq w_{i} \leq w_{M}^{i}, \quad i=1,2, \ldots, n$

where $z_{i}^{*}$ are the optimal values of the optimization problem $\min z_{i}=u_{i}\left(x_{i}^{\mathrm{A}}\right)-u_{i}\left(x_{i}^{\mathrm{C}}\right)$

s.t. $\begin{cases}x_{m i}^{\mathrm{A}} \leq x_{i}^{\mathrm{A}} \leq x_{M i}^{\mathrm{A}}, & i=1,2, \ldots, n \\ x_{m i}^{\mathrm{C}} \leq x_{i}^{\mathrm{C}} \leq x_{M i}^{\mathrm{C}}, & i=1,2, \ldots, n \\ u_{m i}\left(x_{i}^{\mathrm{A}}\right) \leq u_{i}\left(x_{i}^{\mathrm{A}}\right) \leq u_{M i}\left(x_{i}^{\mathrm{A}}\right), & i=1,2, \ldots, n \\ u_{m i}\left(x_{i}^{\mathrm{C}}\right) \leq u_{i}\left(x_{i}^{\mathrm{C}}\right) \leq u_{M i}\left(x_{i}^{\mathrm{C}}\right), & i=1,2, \ldots, n\end{cases}$

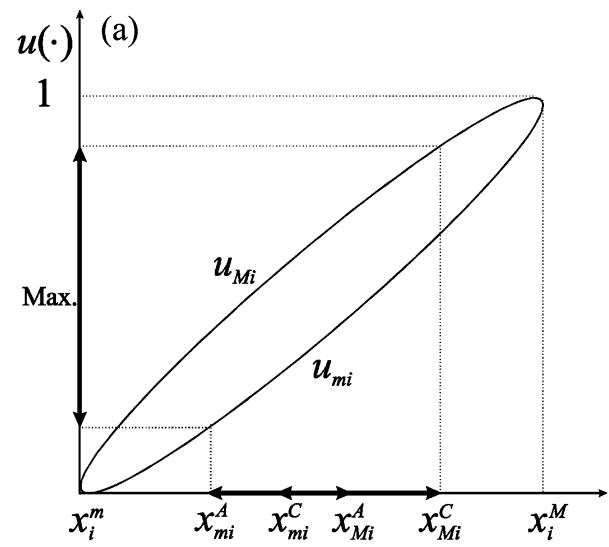

The objective function in problem (12) is

$$
\begin{aligned}
z_{i}= & a_{i j_{1}}+b_{i j_{1}} x_{i}^{\mathrm{A}}+c_{i j_{1}}\left(x_{i}^{\mathrm{A}}\right)^{2}+d_{i j_{1}}\left(x_{i}^{\mathrm{A}}\right)^{3}-\left[a_{i j_{2}}+b_{i j_{2}} x_{i}^{\mathrm{C}}\right. \\
& \left.+c_{i j_{2}}\left(x_{i}^{\mathrm{C}}\right)^{2}+d_{i j_{2}}\left(x_{i}^{\mathrm{C}}\right)^{3}\right]
\end{aligned}
$$

where

$j_{1}=1, j_{2}=1$ if $x_{i}^{\mathrm{A}} \in\left[x_{i}^{m}, x_{i}^{\prime}\right], x_{i}^{\mathrm{C}} \in\left[x_{i}^{m}, x_{i}^{\prime}\right]$

$j_{1}=2, j_{2}=3$ if $x_{i}^{\mathrm{A}} \in\left[x_{i}^{\prime}, x_{i}^{\prime \prime}\right], x_{i}^{\mathrm{C}} \in\left[x_{i}^{\prime \prime}, x_{i}^{\prime \prime \prime}\right]$

$$
\text { : }
$$

The solution to problem (12) can be determined graphically. In fact, let us make a distinction between two cases depending on what the characteristics of the utility function for attribute $i$ are:

(1) If the utility function is increasing, we shall demonstrate that $z_{i}^{*}=u_{m i}\left(x_{m i}^{\mathrm{A}}\right)-u_{M i}\left(x_{M i}^{\mathrm{C}}\right)$.

We prove this assertion by examining the different possibilities that can appear depending on the value intervals for the alternatives. Fig. 3 shows the cases in which each alternative is strictly better than the other for attribute $i$, i.e. it is better for all the possible value combinations in the value intervals (nonoverlapped value intervals).

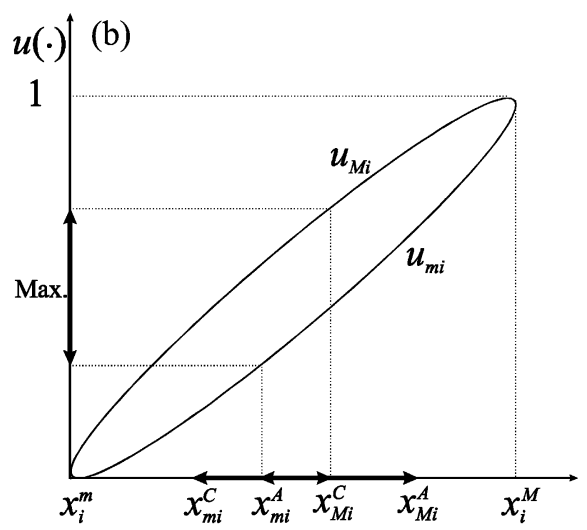

Fig. 4. Overlapped intervals. 

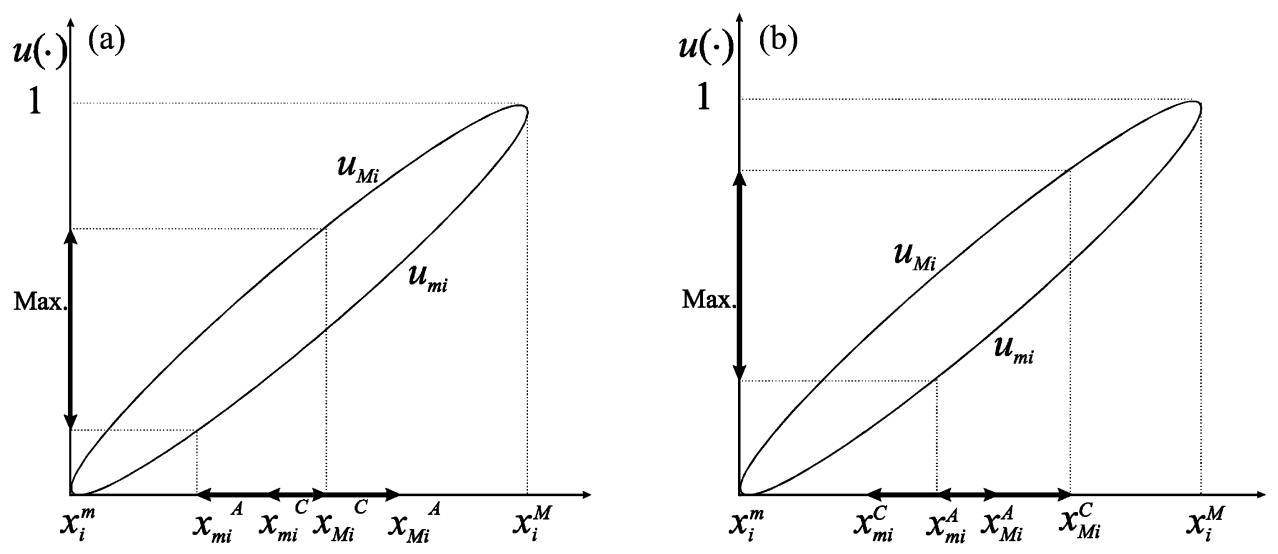

Fig. 5. Contained intervals.

For case (a), in which $S_{\mathrm{C}}$ is better than $S_{\mathrm{A}}$ for the attribute $i$, the difference between the associated utilities, $u_{i}\left(x_{i}^{\mathrm{A}}\right)-u_{i}\left(x_{i}^{\mathrm{C}}\right)$, will be negative and the values $u_{m i}\left(x_{m i}^{\mathrm{A}}\right)$ and $u_{M i}\left(x_{M i}^{\mathrm{C}}\right)$ should be as low as possible, see Eq. (12). In case (b), where $S_{\mathrm{A}}$ is better than $S_{\mathrm{C}}$, the difference between the associated utilities will be positive and again the values $u_{m i}\left(x_{m i}^{\mathrm{A}}\right)$ and $u_{M i}\left(x_{M i}^{\mathrm{C}}\right)$, should be as low as possible.

Below, we examine, the cases where the value intervals for the different alternatives overlap, as shown in Fig. 4.

In this case, there will be combinations of values such that each alternative is better than the other one. As our aim is to minimize the difference between the utilities associated to these values of the alternatives, see Eq. (12), however, we shall retain the values that make $S_{\mathrm{C}}$ better than $S_{\mathrm{A}}$ and that minimize the above-mentioned difference, arriving at the same conclusion as in the cases of nonoverlapped intervals. Finally, we examine the cases in which a value interval is falls within the interval of the other alternative, shown in Fig. 5.

Again, we reach the same conclusions as in the preceding cases.

(2) If the utility function is decreasing $z_{i}^{*}=u_{m i}\left(x_{M i}^{\mathrm{A}}\right)-$ $u_{M i}\left(x_{m i}^{\mathrm{C}}\right)$ holds.

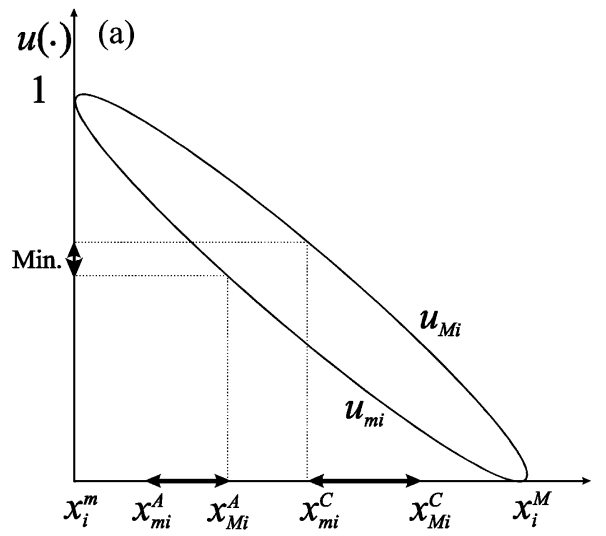

This is demonstrated similarly. Therefore, only the case in which the value intervals of the alternatives are not overlapped is shown here, Fig. 6 .

Having calculated the values $z_{i}^{*}$, they will be substituted in problem (11), which is very easy to solve.

\section{The computation of the potentially optimal alternatives}

The potentially optimal alternatives are alternatives that are possibly optimal for certain values of the weights, certain individual utility functions from the respective class of utility functions and certain values of the consequences of the alternative. From a mathematical point of view, the alternative $S_{j}$ is potentially optimal, if the optimum value of the optimization problem below is smaller than or equal to 0 , $\min f_{j}$

s.t. $\quad \begin{cases}\sum_{i=1}^{n} w_{i} u_{i}\left(x_{i}^{j}\right)-\sum_{i=1}^{n} w_{i} u_{i}\left(x_{i}^{l}\right)+f_{j} \geq 0 & \forall l \neq j \\ x_{m i}^{j} \leq x_{i}^{j} \leq x_{M i}^{j}, & \forall i, j \\ w_{m}^{i} \leq w_{i} \leq w_{M}^{i}, & \forall i \\ u_{m i}\left(x_{i}^{j}\right) \leq u_{i}\left(x_{i}^{j}\right) \leq u_{M i}\left(x_{i}^{j}\right), & \forall i, j\end{cases}$

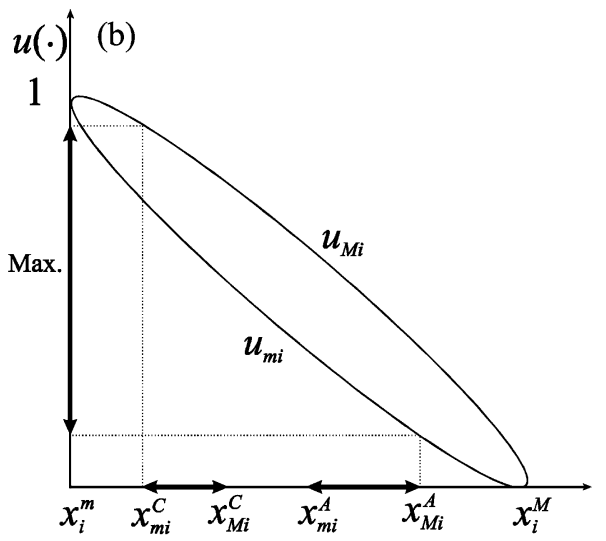

Fig. 6. (a) $S_{\mathrm{C}}$ is preferred to $S_{\mathrm{A}}$; (b) $S_{\mathrm{A}}$ is preferred to $S_{\mathrm{C}}$. 
Note that neither a potentially optimal alternative is necessarily nondominated, nor a nondominated alternative is necessarily potentially optimal. Both concepts are independent. The idea of considering potentially optimal alternatives stems from the fact that can be used to filter the set of alternatives, reducing this set to a size more suitable for study.

Again, we have a nonlinear optimization problem, because some of the constraints are nonlinear. Focusing on the objective function $\min f_{j}$, we see that this term also appears in the earlier constraints. It is obvious that we are looking for as small as possible $f_{j}$. If the constraints of the type $\sum_{i=1}^{n} w_{i} u_{i}\left(x_{i}^{j}\right)-\sum_{i=1}^{n} w_{i} u_{i}\left(x_{i}^{l}\right)+f_{j} \geq 0 \forall l \neq j$ are to be met, $\sum_{i=1}^{n} w_{i} u_{i}\left(x_{i}^{j}\right)-\sum_{i=1}^{n} w_{i} u_{i}\left(x_{i}^{l}\right)$ should have the greatest possible value. Below, we solve the following optimization problem

$\max f_{i}^{j l}=u_{i}\left(x_{i}^{j}\right)-u_{i}\left(x_{i}^{l}\right)$

s.t. $\begin{cases}x_{m i}^{j} \leq x_{i}^{j} \leq x_{M i}^{j}, & i=1,2, \ldots, n \\ x_{m i}^{l} \leq x_{i}^{l} \leq x_{M i}^{l}, & i=1,2, \ldots, n \\ u_{m i}\left(x_{i}^{j}\right) \leq u_{i}\left(x_{i}^{j}\right) \leq u_{M i}\left(x_{i}^{j}\right), & i=1,2, \ldots, n \\ u_{m i}\left(x_{i}^{l}\right) \leq u_{i}\left(x_{i}^{l}\right) \leq u_{M i}\left(x_{i}^{l}\right), & i=1,2, \ldots, n\end{cases}$

Looking at problem (14), we find that it is the same optimization problem as solved for the dominance between alternatives, problem (12), although, in this case, the objective function is for maximization, which merely implies changing the sign of terms in the objective function or considering $\min f_{i}^{l j}$.

After solving $n^{2}-n$ problems of this kind for each $l$, we have a value $f_{i}^{i j *}$ for each solved problem. Moreover, the following constraints will no longer be considered

$x_{m i}^{j} \leq x_{i}^{j} \leq x_{M i}^{j}, \quad i=1,2, \ldots, n$

$x_{m i}^{l} \leq x_{i}^{l} \leq x_{M i}^{l}, \quad i=1,2, \ldots, n$

$u_{m i}\left(x_{i}^{j}\right) \leq u_{i}\left(x_{i}^{j}\right) \leq u_{M i}\left(x_{i}^{j}\right), \quad i=1,2, \ldots, n$

$u_{m i}\left(x_{i}^{l}\right) \leq u_{i}\left(x_{i}^{l}\right) \leq u_{M i}\left(x_{i}^{l}\right), \quad i=1,2, \ldots, n$

because they have already been taken into account in Eq. (14) and the value $f_{i}^{l j *}$ is in this domain.

So, we still have to solve the optimization problem (15). Hence, solving Eq. (13) is equivalent to solving Eqs. (14) and (15).

$\min f_{j}$

s.t. $\quad\left\{\begin{array}{l}\sum_{i=1}^{n} w_{i} f_{i}^{l j *}+f_{j} \geq 0 \quad \forall l \neq j \\ w_{m}^{i} \leq w_{i} \leq w_{M}^{i},\end{array}\right.$

\section{The computation of adjacent potentially optimal alternatives}

Let us denote the optimal alternative in the ranking obtained from considering the midpoints of the utility interval of each alternative as $S^{*}$, which would be equivalent to a consideration of precision. An alternative is adjacent potentially optimal if it can share optimality with $S^{*}$.

To find out whether an alternative $S_{j}$ is adjacent potentially optimal to the optimal $S^{*}$, we have to solve the optimization problem:

$$
\begin{array}{ll}
\min g_{j}= & \left|\sum_{i=1}^{n} w_{i} u_{i}\left(x_{i}^{j}\right)-\sum_{i=1}^{n} w_{i} u_{i}\left(x_{i}^{*}\right)\right| \\
\text { s.t. } \quad \begin{cases}\sum_{i=1}^{n} w_{i} u_{i}\left(x_{i}^{l}\right)-\sum_{i=1}^{n} w_{i} u_{i}\left(x_{i}^{*}\right) \leq 0 & \forall l \neq j \\
x_{m i}^{j} \leq x_{i}^{j} \leq x_{M i}^{j}, & \forall i, j \\
w_{m}^{i} \leq w_{i} \leq w_{M}^{i}, & \forall i \\
u_{m i}\left(x_{i}^{j}\right) \leq u_{i}\left(x_{i}^{j}\right) \leq u_{M i}\left(x_{i}^{j}\right), & \forall i, j\end{cases}
\end{array}
$$

After solving the problem, if the optimum value $g_{j}^{*}=0$, we say that $S_{j}$ is adjacent potentially optimal to the optimal alternative.

Looking at problem (16), it appears that the solutions domain is the same as the one used for the dominance problem, although constraints of the kind $\sum_{i=1}^{n} w_{i} u_{i}\left(x_{i}^{l}\right)-$ $\sum_{i=1}^{n} w_{i} u_{i}\left(x_{i}^{*}\right) \leq 0, \forall l \neq j$ are added, and only the alternatives that have passed through the potentially optimal filter are taken as alternatives $S_{l}$. Furthermore, this kind of constraint is familiar to us, because $\sum_{i=1}^{n} w_{i} u_{i}\left(x_{i}^{l}\right)-\sum_{i=1}^{n} \times$ $w_{i} u_{i}\left(x_{i}^{*}\right)$ is the expression of the objective function of the dominance problems of $S^{*}$ with respect to $S_{l}$.

Taking the dominance problem (9) of $S^{*}$ with respect to $S_{l}$, we have

$$
\begin{aligned}
& \min f_{* l}=\sum_{i=1}^{n} w_{i} u_{i}\left(x_{i}^{l}\right)-\sum_{i=1}^{n} w_{i} u_{i}\left(x_{i}^{*}\right) \\
& \text { s.t. } \quad \begin{cases}x_{m i}^{l} \leq x_{i}^{l} \leq x_{M i}^{l}, & i=1,2, \ldots, n \\
x_{m i}^{*} \leq x^{*} \leq x_{M i}^{*}, & i=1,2, \ldots, n \\
w_{m}^{i} \leq w_{i} \leq w_{M}^{i}, & i=1,2, \ldots, n \\
u_{m i}\left(x_{i}^{l}\right) \leq u_{i}\left(x_{i}^{l}\right) \leq u_{M i}\left(x_{i}^{l}\right), & i=1,2, \ldots, n \\
u_{m i}\left(x_{i}^{*}\right) \leq u_{i}\left(x_{i}^{*}\right) \leq u_{M i}\left(x_{i}^{*}\right), & i=1,2, \ldots, n\end{cases}
\end{aligned}
$$

We know beforehand that $f_{* l}^{*}$ is smaller than or equal to 0 , because the alternative $S^{*}$ is the optimal alternative and we have a feasible solution, which meets the constraint $\sum_{i=1}^{n} \times$ $w_{i} u_{i}\left(x_{i}^{l}\right)-\sum_{i=1}^{n} w_{i} u_{i}\left(x_{i}^{*}\right) \leq 0$ and also verifies the constraints of problem (17).

Although the weights constraint $w_{m}^{i} \leq w_{i} \leq w_{M}^{i}, i=$ $1,2, \ldots, n$, is met we are only interested at this point in fixing the variables that verify

$$
\begin{aligned}
& x_{m i}^{l} \leq x_{i}^{l} \leq x_{M i}^{l}, \quad i=1,2, \ldots, n \\
& x_{m i}^{*} \leq x^{*} \leq x_{M i}^{*}, \quad i=1,2, \ldots, n \\
& u_{m i}\left(x_{i}^{l}\right) \leq u_{i}\left(x_{i}^{l}\right) \leq u_{M i}\left(x_{i}^{l}\right), \quad i=1,2, \ldots, n \\
& u_{m i}\left(x_{i}^{*}\right) \leq u_{i}\left(x_{i}^{*}\right) \leq u_{M i}\left(x_{i}^{*}\right), \quad i=1,2, \ldots, n
\end{aligned}
$$




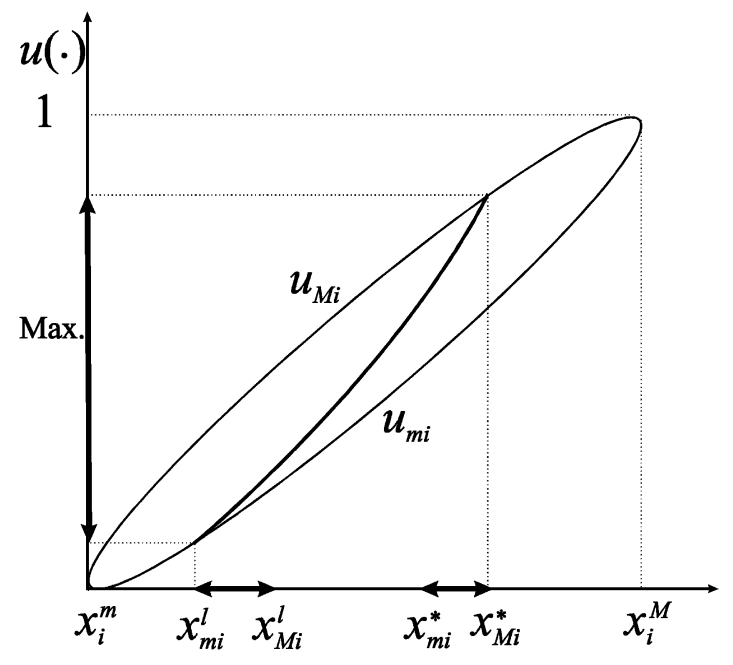

Fig. 7. Assignment of the utility function.

Throughout problem solving Eq. (17), we look for a feasible solution that provides the values of the individual utility functions inside of the respective classes, i.e. we calculate the values of the variables $a_{i j}, b_{i j}, c_{i j}$ and $d_{i j}$, with $j=1-4$, Fig. 7.

Fig. 7 shows how the value of the individual utility function is fixed taking into account the values for alternatives $S_{l}$ and $S^{*}$ on the attribute $i$ for the dominance problem of $S^{*}$ with respect to $S_{l}$. As we want to minimize the difference between utilities, we take the minimum and the maximum expected utilities $x_{m i}^{l}$ and $x_{M i}^{*}$, respectively, and by means of an interpolation using these points, we get the expression of the cubic spline by intervals, which represents the individual utility function of this attribute.

The reason why we are not interested in fixing the values for the weights is fixing all the variables will guarantee that the constraints $\sum_{i=1}^{n} w_{i} u_{i}\left(x_{i}^{k}\right)-\sum_{i=1}^{n} w_{i} u_{i}\left(x_{i}^{*}\right) \leq 0 \quad \forall k \neq$ $j, l$ are met. If we do not fix the weights in the first step, we can get for each additional constraint $\sum_{i=1}^{n} w_{i} u_{i}\left(x_{i}^{k}\right)-$ $\sum_{i=1}^{n} w_{i} u_{i}\left(x_{i}^{*}\right) \leq 0 \forall k \neq j, l$, a set of precise individual utility functions in the different attributes of the hierarchy. Now, each one of these founded feasible solutions to the problem, will be substituted in problem (16) to obtain a feasible solution.

At this point, we have an almost linear problem, because of the absolute value in the objective function, and it will be necessary to consider a transformation. Our problem (16) now has the following form

$\min g_{j}=\left|u\left(S_{j}, w\right)-u\left(S^{*}, w\right)\right|$

s.t. $\quad \begin{cases}u\left(S_{l}, w\right)-u\left(S^{*}, w\right) \leq 0 & \forall l \neq j \\ w_{m}^{i} \leq w_{i} \leq w_{M}^{i}, & i=1,2, \ldots, n\end{cases}$

We use the expression $u\left(S_{j}, w\right)-u\left(S^{*}, w\right)$ to indicate that the objective function depends only on the variables weight.

Problem (18) is equivalent to the linear problem $\min t$

s.t. $\begin{cases}u\left(S_{l}, w\right)-u\left(S^{*}, w\right) \leq 0 & \forall l \neq j \\ w_{m}^{i} \leq w_{i} \leq w_{M}^{i}, & i=1,2, \ldots, n \\ -t \leq u\left(S_{j}, w\right)-u\left(S^{*}, w\right) \leq t & \end{cases}$

Note that because we have not fixed the constraints for the weights in the first stage, in the second stage, when fixing the weights, the best solution is kept inside the family of the feasible solutions that have defined the same values for the rest of variables during the second stage.

Following this process for each one of the constraints of the kind

$\sum_{i=1}^{n} w_{i} u_{i}\left(x_{i}^{k}\right)-\sum_{i=1}^{n} w_{i} u_{i}\left(x_{i}^{*}\right) \leq 0$

we finally obtain a set of possible solutions $S_{1}, \ldots, S_{r}$. But we have to retain the best one. We substitute each solution in the objective of problem (16) $g_{j}=\left|\sum_{i=1}^{n} w_{i} u_{i}\left(x_{i}^{j}\right)-\sum_{i=1}^{n} w_{i} u_{i}\left(x_{i}^{*}\right)\right|$, and the optimal solution $S_{j}$ will be the solution with the lowest value.

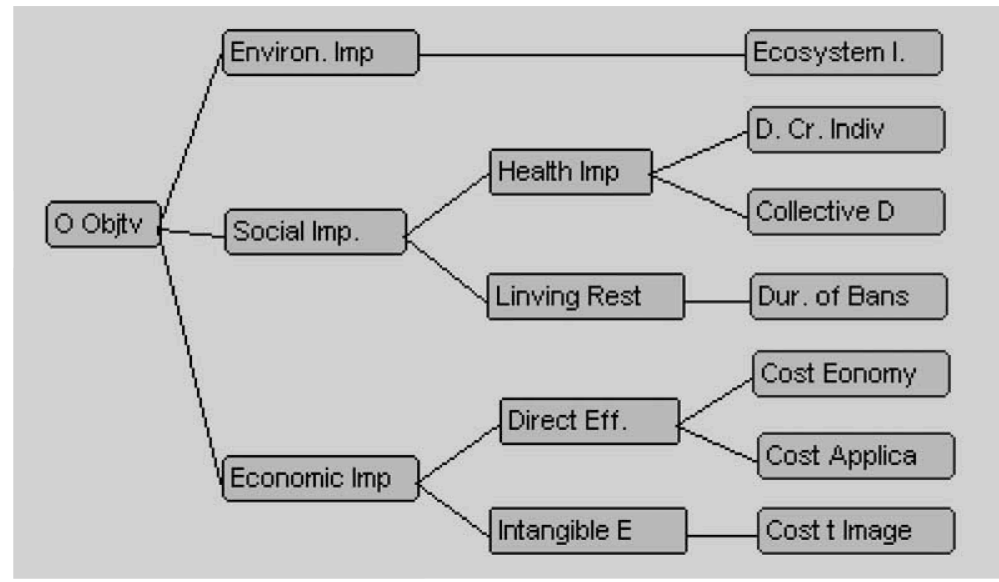

Fig. 8. Objectives hierarchy for lake Øvre Heimdalsvatn. 


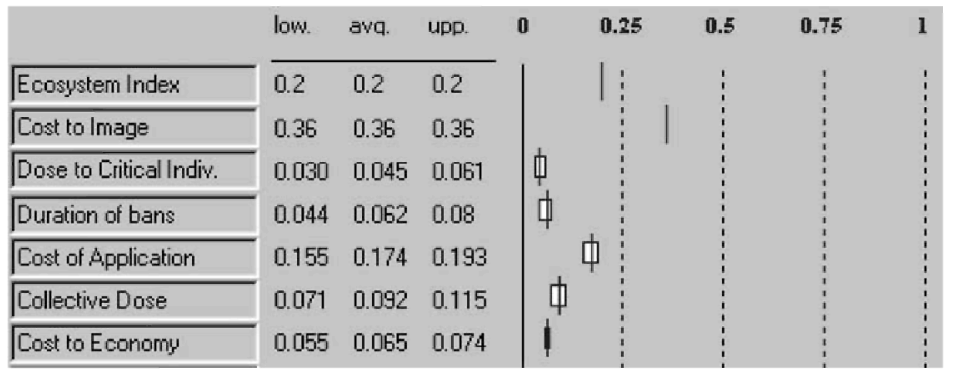

Fig. 9. Attributes imprecise weights.

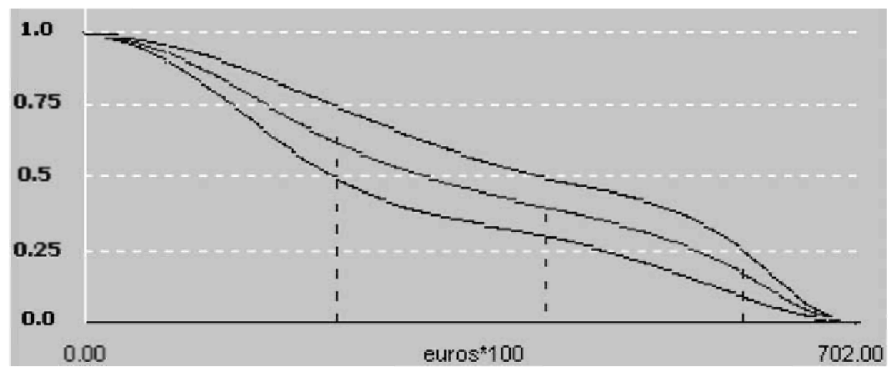

Fig. 10. Class of utility functions for the attribute Cost to Application.

\section{Example}

We illustrate the optimization problems with an application example based on our implemented Decision Support System. The considered scenario is lake $\emptyset_{\mathrm{vre}}$ Heimdalsvatn, located in Oppland county (Norway). This lake has been thoroughly studied [17].

After the Chernobyl accident, the lake was contaminated with a fallout of $130 \mathrm{kBq} / \mathrm{m}^{2}$ of ${ }^{137} \mathrm{Cs}$, which, in principle, required no countermeasures, as a result of since the lake is not much used by human beings. However, an evaluation of alternative strategies was made for the purpose of testing the MOIRA system [4].

For the particular case under study, the objectives hierarchy is as shown in Fig. 8.

The system admits imprecise assignments for weights and utilities, Figs. 9 and 10, and uncertainty in the multiattribute strategies, which can be defined in terms of ranges for each attribute instead of single values.

In our example, a set of nine strategies were analysed, combining chemical countermeasures with bans on fishing so as to reduce the radiological and environmental impact, Table 1. Observe that the first attribute, $X_{1}$, corresponds to 'ecosystem index', Fig. 8, and so on, being the last 'cost of image' associated to $X_{7}$.

We can rank the strategies, obtaining the results shown in Fig. 11.

In Fig. 12 we show the nondominated, potentially optimal, adjacent potentially optimal strategies.

Observe that, from the nine initial strategies, three are nondominated (the set of strategies of interest has been considerably reduced), which are also potentially optimal and 'L.Liming + Fish Bans (3)' and 'Fertilization + Fish Bans (3)' are adjacent optimal to 'Fertilization', which is the best ranked.

Table 1

Strategies consequences

\begin{tabular}{|c|c|c|c|c|c|c|c|}
\hline \multirow[t]{2}{*}{ Strategies } & \multicolumn{7}{|l|}{ Attributes } \\
\hline & $X_{1}$ & $X_{2}$ & $X_{3}$ & $X_{4}$ & $X_{5}$ & $X_{6}$ & $X_{7}$ \\
\hline No action & {$[5.0,5.0]$} & {$[2.2,2.4]$} & {$[64,72]$} & {$[0,6]$} & {$[0,0]$} & {$[0,0]$} & {$[0.0,0.0]$} \\
\hline Fish Bans (1st) & {$[4.0,5.0]$} & {$[1.8,2.3]$} & {$[56,65]$} & {$[0,10]$} & {$[130,170]$} & {$[0,25]$} & {$[0.0,0.0]$} \\
\hline Fish Bans (2-4) & {$[3.5,5.0]$} & {$[0.8,1.3]$} & {$[21,27]$} & {$[30,36]$} & {$[380,426]$} & {$[10,50]$} & {$[0.0,0.0]$} \\
\hline Lake Liming & {$[1.5,2.5]$} & {$[2.2,2.4]$} & {$[61,70]$} & {$[0,6]$} & {$[0,40]$} & {$[130,190]$} & {$[0.7,0.7]$} \\
\hline Liming + F.Bans (3) & {$[1.5,2.6]$} & {$[0.8,1.3]$} & {$[21,29]$} & {$[30,36]$} & {$[390,426]$} & {$[110,160]$} & {$[0.7,0.7]$} \\
\hline Potash Treatment & {$[1.8,2.7]$} & {$[1.9,2.3]$} & {$[56,61]$} & {$[0,5]$} & {$[0,35]$} & {$[610,702]$} & {$[0.6,0.6]$} \\
\hline Potash + F.Bans (3) & {$[1.8,2.6]$} & {$[0.7,1.3]$} & {$[20,29]$} & {$[32,36]$} & {$[406,426]$} & {$[665,702]$} & {$[0.6,0.6]$} \\
\hline Fertilization & {$[1.0,1.6]$} & {$[2.0,2.4]$} & {$[65,72]$} & {$[0,3]$} & {$[0,35]$} & {$[112,168]$} & {$[1.0,1.0]$} \\
\hline Fertiliz + F.Bans (3) & {$[1.0,1.8]$} & {$[0.7,1.4]$} & {$[20,29]$} & {$[34,36]$} & {$[420,426]$} & {$[110,155]$} & {$[1.0,1.0]$} \\
\hline
\end{tabular}




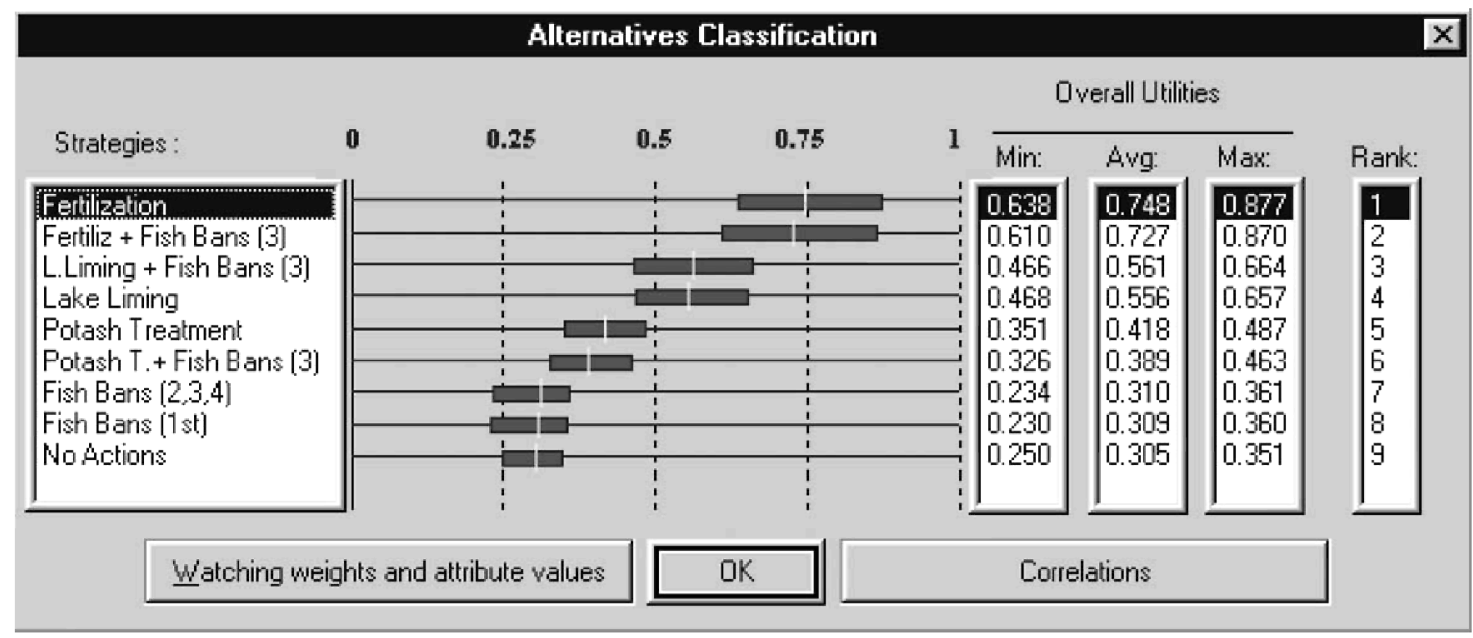

Fig. 11. Graphical representation of utilities.

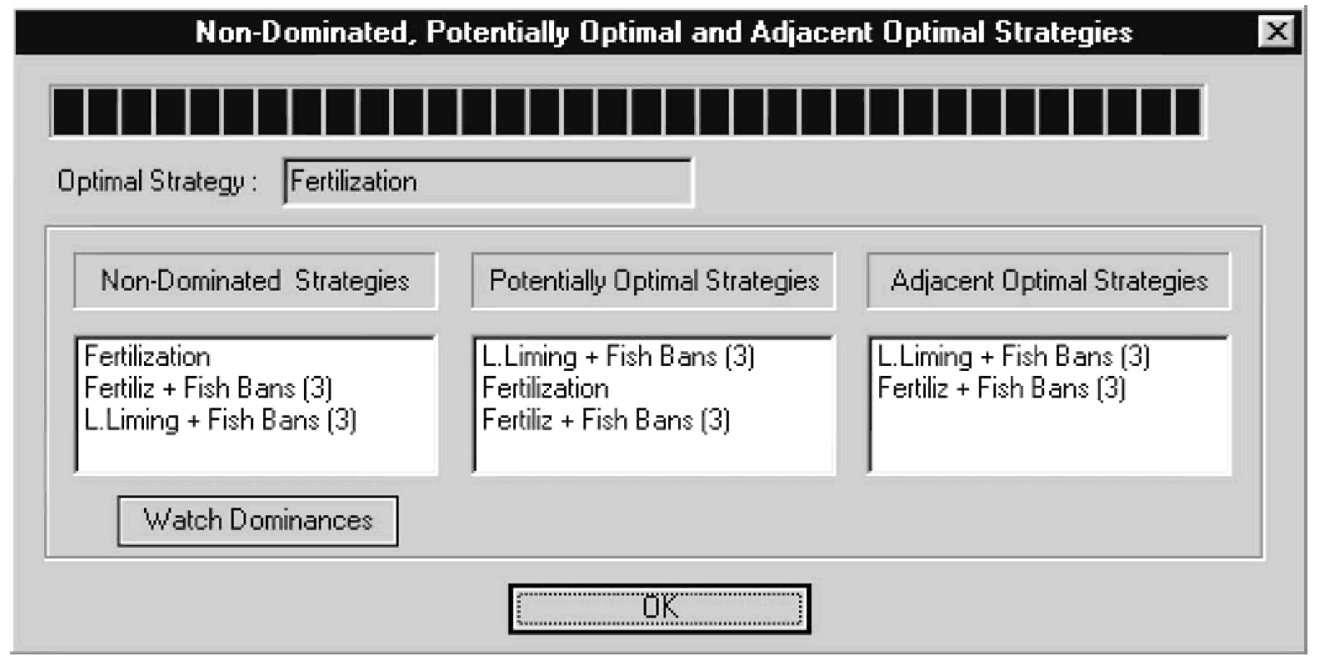

Fig. 12. Nondominated, potentially optimal, adjacent potentially optimal strategies.

Finally, let us emphasize that if we had not compute the nondominated strategies, and just we should have the summary results of Fig. 11, it would be impossible to discover that the strategy 'lake liming' is dominated and for the DM should never be the optimum.

\section{Conclusions}

The optimization problems to be solved in multicriteria decision-making to determine the nondominated, potentially optimal and adjacent potentially optimal alternatives to the optimal alternative are nonlinear if we assume that there is imprecision in the consequences of the alternatives and in DM preferences. However, if such preferences can be represented by means of an additive multiattribute utility function, where the component utility functions are cubic splines, the problems in computing the nondominated and potentially optimal alternatives can be solved by means of a sequence of linear optimization problems. This approach is not directly applicable for adjacent potentially optimal alternatives, but we have proposed a heuristic, which is an important aid in their computation.

\section{Acknowledgements}

This paper was supported by the Ministry of Science and Technology project DPI2001-3731, the U.P.M. Project A9912 and the Madrid regional government project CAM 07T/0027/2000. 


\section{References}

[1] Raiffa H. Decision analysis. Introductory lectures on choices under uncertainty, Reading, MA: Addison-Wesley; 1968.

[2] French S. Decision theory: an introduction to the mathematics of rationality. Chichester: Horwood; 1986.

[3] Keeney RL, Raiffa H. Decision with multiple objectives: preferences and value-tradeoffs. Cambridge: Cambridge University Press; 1993.

[4] Ríos Insua D, Gallego E, Mateos A, Ríos-Insua S. MOIRA: a decision support system for decision making on aquatic ecosystem contaminated by radioactive fallout. Ann Oper Res 2000;95:341-64.

[5] Mateos A, Ríos-Insua S, Gallego E. Postoptimal analysis in a multiattribute decision model for restoring contaminated aquatic ecosystems. J Oper Res Soc 2001;52:1-12.

[6] Brownlow SA, Watson SR. Structuring multi-attribute value hierarchies. J Oper Res Soc 1987;38:309-17.

[7] Raiffa H. The art and science of negotiation. Cambridge, MA: Harvard University Press; 1982.

[8] Stewart TJ. Robustness of additive value function method in MCDM. J Multi-Criteria Decision Anal 1996;5:301-9.

[9] von Nitzsch R, Weber M. Utility function assessment on a microcomputer: an interactive procedure. Ann Oper Res 1998;16:149-60.
[10] Ríos S, Ríos-Insua S, Ríos Insua D, Pachón J. Experiments in robust decision making. In: Ríos $S$, editor. Decision theory and decision analysis, trends and challenges. Boston: Kluwer; 1994. p. $233-42$.

[11] Farquhar PH. Utility assessment methods. Mgmt Sci 1984;30: $1283-300$

[12] Gómez M, Ríos-Insua S, Bielza C, Fdez del Pozo JA. Multi-attribute utility analysis in the IctNeo system. In: Haimes YY, Steuer R, editors. Research and practice in multiple criteria decision making. LNEMS 487, Berlin: Springer; 2000. p. 81-92.

[13] Hazen GB. Partial information, dominance and potential optimality in multi-attribute utility theory. Oper Res 1986;34:296-310.

[14] Ríos Insua D. Sensitivity analysis multiobjetive decision making. LNEMS 347, Berlin: Springer; 1990.

[15] Ríos Insua D, French S. A framework for sensitivity analysis in discrete multi-objective decision-making. Eur J Oper Res 1991;54: $176-90$.

[16] Proll LG, Salhi A, Ríos Insua D. Improving an optimization based framework for sensitivity analysis in multi-criteria decision-making. J Multi-Criteria Decision Anal 2001;10:1-9.

[17] Vik R. The Lake Øvre Heimdalsvatn, a Subalpine Freshwater Ecosystem. Holartic Ecol 1978;1:81-320. 